\title{
Use of Mobile Health (mHealth) Tools by Primary Care Patients in the WWAMI Region Practice and Research Network (WPRN)
}

\author{
Amy M. Bauer, MD, MS, Tessa Rue, PhD, Gina A. Keppel, MPH, \\ Allison M. Cole, MD, MPH, Laura-Mae Baldwin, MD, and Wayne Katon, MD
}

Purpose: The purpose of this study was to determine the prevalence of mobile health (mHealth) use among primary care patients and examine demographic and clinical correlates.

Metbods: Adult patients who presented to 1 of 6 primary care clinics in a practice-based research network in the northwest United States during a 2-week period received a survey that assessed smartphone ownership; mHealth use; sociodemographic characteristics (age, sex, race/ethnicity, health literacy); chronic conditions; and depressive symptoms (2-item Patient Health Questionnaire). Data analysis used descriptive statistics and mixed logistic regression.

Results: Of 918 respondents (estimated response rate, 67.4\%), 55\% owned a smartphone, among whom $70 \%$ were mHealth users. In multivariate analyses, smartphone ownership and mHealth use were not associated with health literacy, chronic conditions, or depression but were less common among adults $>45$ years old (adjusted odds ratio, $0.07-0.39 ; P<.001$ ). Only $10 \%$ of patients learned about mHealth tools from their physician, and few (31\%) prioritized their provider's involvement.

Conclusions: Use of mHealth technologies is lower among older adults but otherwise is common among primary care patients, including those with limited health literacy and those with chronic conditions. Findings support the potential role of mHealth in improving disease management among certain groups in need; however, greater involvement of health care providers may be important for realizing this potential. (J Am Board Fam Med 2014;27:780-788.)

Keywords: Chronic Disease, Mobile Health, Practice-based Research, Primary Health Care

Mobile health (mHealth), which refers to the application of mobile or wireless communication technologies to health and health care, ${ }^{1}$ barely ex-

This article was externally peer reviewed.

Submitted 31 March 2014; revised 14 August 2014; accepted 18 August 2014.

From the Department of Psychiatry and Behavioral Sciences (AMB, WK), the Department of Biostatistics (TR), the Institute of Translational Health Sciences (TR, GAK, AMC), and the Department of Family Medicine (GAK, AMC, L-MB), University of Washington, Seattle.

Funding: This project was supported by the National Center for Advancing Translational Sciences of the National Institutes of Health under award no. KL2TR000421 and grant UL1TR000423 through the Clinical and Translational Science Awards (CTSA) Program.

Conflict of interest: none declared.

Disclaimer: The content of this article is solely the responsibility of the authors and does not necessarily represent the official views of the National Institutes of Health.

Corresponding author: Amy M. Bauer, MD, MS, Department of Psychiatry and Behavioral Sciences, University of Washington School of Medicine, 1959 NE Pacific Street, Box 356560, Seattle, WA 98195-6560 (E-mail: abauer1@uw.edu). isted 5 years ago; it has since emerged as a multibillion-dollar industry. ${ }^{2}$ Recent data suggest that $>90,000$ consumer smartphone health applications ("apps") are now available for download. ${ }^{3}$ Few of these have been the subject of scientific study, including their potential risks, ${ }^{4}$ or to US Food and Drug Administration review or approval. ${ }^{1}$

According to estimates from the Pew Internet \& American Life Project, in 2012 nearly one third of mobile phone owners, and more than half of smartphone owners, have used their phone to look up health information-a rate that has nearly doubled since 2010. In Pew's general population survey, mHealth use was more common among individuals $<50$ years old, Latinos, African Americans, and those with higher socioeconomic status.

The potential for mHealth tools to represent an important advancement in health care, in particular for chronic disease care, has been recognized. ${ }^{1}$ 
Whereas an estimated $69 \%$ of the US adult population track at least 1 health indicator, such as activity, weight, or symptoms, only about $20 \%$ of those use technology to facilitate their tracking. ${ }^{6}$ Although health tracking is significantly more common among people living with chronic conditions, mHealth use has been reported as significantly less common among these individuals. ${ }^{5,6}$ Primary care is the setting in which most chronic disease care occurs, and although there some literature describes mHealth tools available to facilitate chronic disease management, the actual patterns of mHealth use among patients in primary care settings have not been documented. ${ }^{7-9}$ To address this gap in knowledge, this study aimed to determine the prevalence of mobile phone ownership and mHealth use among primary care patients in community-based clinics in the WWAMI region Practice and Research Network (WPRN) and to examine demographic and clinical correlates of use.

\section{Methods \\ Setting}

The WPRN is a primary care practice-based research network in the 5-state WWAMI region: Washington, Wyoming, Alaska, Montana, and Idaho. The 6 sites that participated in the study came from 4 of these states (Washington, Wyoming, Alaska, and Idaho) and included 4 hospitalassociated outpatient practices, 1 office practice, and 1 federally qualified health center. The clinics serve many low-income patients; the proportion of patients who are uninsured or receiving Medicaid ranges from $22 \%$ to $62 \%$. A champion at each clinic site, who had the opportunity to provide feedback on the survey design, assisted in study coordination, facilitated implementation of the study procedures, and returned completed surveys to the investigators, was engaged throughout the project. Participating sites received an administrative stipend of $\$ 500$.

\section{Participants and Procedures}

This study used a card study method, an established means of collecting data in primary care settings, described by Westfall and colleagues ${ }^{10}$ as a methodology used to conduct a prevalence study by collecting data from patients who consecutively present to a clinic. All adult patients ( $\geq 18$ years old) seen for a visit in one of the participating sites during a 2-week period in June 2013 were eligible to participate. When checking in for their appointments at the front desk, patients were given a 1-page questionnaire designed to be completed in $<5$ minutes. The questionnaire was anonymous, and patients were informed that participation was voluntary and would not affect their health care. Participants returned the surveys to a collection box in the waiting area to ensure anonymity. Each questionnaire had a unique tracking number that was used to facilitate estimation of the response rate. Each day, front desk staff recorded the tracking number of the first and last questionnaire distributed for the day. If a participant declined to complete the questionnaire, the questionnaire form was discarded or placed in the collection box blank to avoid reuse. Questionnaires that were not distributed to patients were returned to the investigators after the completion of data collection. This study was considered minimal risk and was granted an exempt determination by the institutional review board at the University of Washington.

\section{Measures}

\section{Dependent Variables}

Two questions were adapted from the Pew Internet \& American Life project to assess mobile phone and smartphone ownership. ${ }^{5}$ Mobile health use was assessed by asking participants if they have ever used their phone to "find health or medical information," "download or use a health 'app'," or "track or manage a health issue (your diet or weight, activity, mood, blood pressure, etc.)." Participants were coded as mHealth users if they reported at least one of these uses and endorsed both mobile and smartphone ownership. Mobile health users indicated the frequency of use and completed 5 additional items about their mHealth use. The questionnaire included skip logic so that participants who did not endorse mobile phone ownership were not asked about smartphone ownership or mHealth use and were coded as "no" for these items. Participants who did not endorse smartphone ownership were not asked about mHealth use and were coded as not using mHealth tools. Regardless of phone ownership or mHealth use, all participants were asked if they use an Internet patient portal, how useful they believe receiving health materials from their provider on their phone would be, and their comfort with the security of 
health information that is shared with their doctor or stored by a third party.

\section{Independent Variables}

Demographic information included participants' age, sex, and self-reported race/ethnicity. Patients reported whether they had any of the following common chronic medical conditions: high blood pressure, diabetes, heart disease, asthma, chronic pain, depression, or any other chronic condition not listed. The 2-item Patient Health Questionnaire was used to assess the level of current depressive symptoms. Scores on the Patient Health Questionnaire range from 0 to 6 , and a cutpoint of 3 has been used to identify patients with probable major depression, with high sensitivity and specificity. ${ }^{11,12}$ Health literacy was assessed with 3-item instrument that has been validated against established measures of health literacy in a variety of settings. ${ }^{13-15}$ Each item was scored from 1 to 5, and the scores were summed to yield a total score ranging from 3 (poor health literacy) to 15 (no health literacy limitations). Consistent with prior research, scores were coded dichotomously as having no health literacy limitations (score of 15) versus any limitation (scores of 3-14). ${ }^{16,17}$

\section{Data Analysis}

Descriptive statistics, including frequencies, means, and standard deviations, were used to characterize patients' demographic and clinical features, mobile and smartphone ownership, and mHealth use. Associations between patient characteristics and technology ownership and use were examined with both unadjusted and adjusted mixed logistic regression models, clustered by clinic site. Specifically, separate mixed effects logistic regression models estimated the unadjusted associations between each patient characteristic (age, sex, race/ethnicity, health literacy, chronic conditions, and depression) and each dependent variable (smartphone ownership and mHealth use). Subsequently, for each dependent variable, a multivariate mixed logistic regression models was specified; all patient variables were entered simultaneously to estimate the independent associations between patient characteristics and smartphone ownership and use after adjusting for the effect of the remaining patient covariates. The primary analyses were conducted on the full cohort of patients in the sample. In sensitivity analyses, the regression models included patient cohorts that
Table 1. Demographic and Clinical Characteristics of Patients Sampled from 6 Primary Care Practices in Washington, Wyoming, Alaska, and Idaho (June 2013)

\begin{tabular}{lc}
\hline Characteristics & Patients, $\mathrm{n}(\%)$ \\
\hline Age, years $(\mathrm{n}=858)$ & \\
$18-24$ & $130(15.2)$ \\
$25-34$ & $183(21.3)$ \\
$35-44$ & $164(19.1)$ \\
$45-54$ & $163(19.0)$ \\
$55-64$ & $140(16.3)$ \\
$\geq 65$ & $78(9.1)$ \\
Female sex (n = 859) & $643(74.9)$ \\
Race/ethnicity (n $=867)$ & \\
White & $694(80.1)$ \\
Native American & $18(2.1)$ \\
Asian/Pacific Islander & $31(3.6)$ \\
African American & $21(2.4)$ \\
Latino & $42(4.8)$ \\
Other/multiracial & $61(7.0)$ \\
Any health literacy limitation $(\mathrm{n}=844)$ & $526(62.3)$ \\
Any chronic medical condition $(\mathrm{n}=909)$ & $575(63.3)$ \\
Depression (n = 808)* & $334(41.3)$ \\
\hline
\end{tabular}

*Depression is defined as a 2-item Patient Health Questionnaire score $\geq 3$ or a history of depression endorsed as a medical condition.

were constructed based on the questionnaire skip logic (ie, regression models predicting smartphone ownership were restricted to mobile phone owners and models predicting mHealth use were restricted to smartphone owners). Multiple imputation ( $\mathrm{m}=$ 40) was used to impute missing independent variables in multivariate models using "mi" commands in Stata software (StataCorp, College Station, TX). All variables were included in the imputation and assumed to have a multivariate normal distribution. Multivariate regressions were performed on each imputed data set, and results were combined using the rules defined by Rubin. ${ }^{18}$

\section{Results}

Based on the tracking procedures, an estimated 1363 questionnaires were distributed and 918 were completed, for an estimated response rate of $67.4 \%$ (range, $31.8-84.0 \%$ across sites). Participants varied considerably in age; most $(62 \%)$ had some health literacy limitations, most $(63 \%)$ reported at least 1 chronic condition, and many (41\%) reported current or prior depression (Table 1). As depicted in Figure 1, nearly all patients $(91.0 \%, \mathrm{n}=835)$ 
Figure 1. Mobile phone ownership and mHealth use among primary care patients.

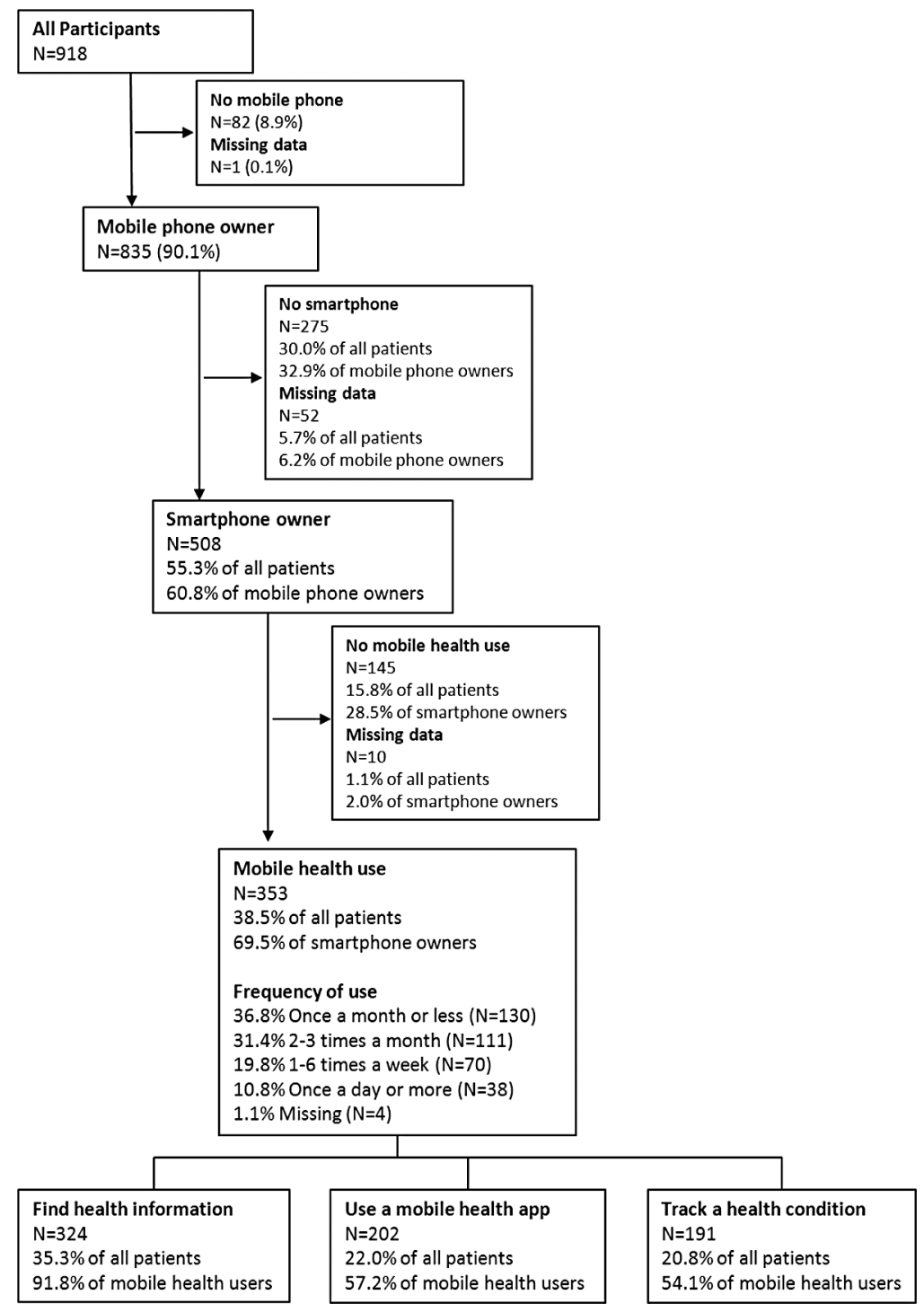

owned a mobile phone and a majority owned a smartphone $(55.3 \%$ of all patients $[\mathrm{n}=508]$ or $60.8 \%$ of mobile phone owners). Almost $70 \%$ ( $\mathrm{n}=$ 353) of smartphone owners reported mHealth use, which corresponds to $38.5 \%$ of all patients surveyed. Nearly all mHealth users reported using their phones to find health or medical information $(91.8 \%, \mathrm{n}=324)$. A majority also reported downloading or using a health app $(57.2 \%, \mathrm{n}=202)$ or using their phone to track or manage a health condition $(54.1 \%, \mathrm{n}=191)$. As shown in Table 2, most patients who used mHealth tools did so infrequently: $69.1 \%(\mathrm{n}=241)$ reported use $\leq 3$ times per month, whereas $10.8 \%(\mathrm{n}=38)$ reported use on a daily basis.

\section{Types of Mobile Health Use}

Among 353 mHealth users, over one-third listed a general health app such as WebMD, iTriage, or Mayo Clinic as their favorite app (Table 2). The next most popular types were activity or fitness apps and weight loss or diet apps, which together were listed as the favorite by one quarter of the sample. Apps for chronic disease management, including those that monitor glucose or mood, were cited as a favorite by only 6 patients $(2.6 \%$ of responses). Many respondents (30.4\%) reported using an app for a short period of time then stopping, most frequently $(48.0 \%)$ because it was too time-consuming. Patients rated appointment reminders as the most useful potential feature, fol- 
Table 2. Description of mHealth Use Among Primary Care Patients Who Report Such Use $(n=353)$

\begin{tabular}{|c|c|}
\hline mHealth Use & $\begin{array}{c}\text { Primary Care } \\
\text { Patients } \\
\text { Reporting Use }\end{array}$ \\
\hline \multicolumn{2}{|l|}{ Mobile health use and type } \\
\hline Find health information & $324(91.8)$ \\
\hline Use health apps & $202(57.2)$ \\
\hline Track or manage health condition & $191(54.1)$ \\
\hline \multicolumn{2}{|l|}{ Frequency of mHealth use $(\mathrm{n}=349)$} \\
\hline Once a month or less & $130(37.3)$ \\
\hline $2-3$ times a month & $111(31.8)$ \\
\hline 1-6 times a week & $70(20.1)$ \\
\hline Once a day or more & $38(10.9)$ \\
\hline \multicolumn{2}{|l|}{ Favorite app $(\mathrm{n}=235)$} \\
\hline $\begin{array}{l}\text { General health app (eg, WedMD, iTriage, } \\
\text { Mayo Clinic) }\end{array}$ & $85(36.2)$ \\
\hline Activity/fitness (eg, My Fitness Pal) & $34(14.5)$ \\
\hline $\begin{array}{l}\text { Weight/diet (eg, Weight Watchers, } \\
\text { LoseIt) }\end{array}$ & $24(10.2)$ \\
\hline $\begin{array}{l}\text { Reproductive (menstrual, pregnancy, or } \\
\text { infant trackers) }\end{array}$ & $19(8.1)$ \\
\hline Web search (eg, Google, Bing) & $18(7.7)$ \\
\hline More than one favorite app & $18(7.7)$ \\
\hline Other (eg, goal trackers, smoking logs) & $14(6.0)$ \\
\hline Patient portal (eg, e-care, MyChart) & $10(4.3)$ \\
\hline $\begin{array}{l}\text { Medication (eg, pharmacy app, pill } \\
\text { trackers) }\end{array}$ & $7(3.0)$ \\
\hline $\begin{array}{l}\text { Disease specific (eg, glucose monitoring, } \\
\text { mood monitoring) }\end{array}$ & $6(2.6)$ \\
\hline $\begin{array}{l}\text { Ever stopped using an app after a short time } \\
\quad(\mathrm{n}=310)\end{array}$ & $122(39.4)$ \\
\hline \multicolumn{2}{|l|}{$\begin{array}{l}\text { Reasons cited for stopping use of an app } \\
\text { after a short time }(\mathrm{n}=98)\end{array}$} \\
\hline Took too much time & $47(48.0)$ \\
\hline Didn't do what you wanted & $32(32.7)$ \\
\hline Problem with login/password & $6(6.1)$ \\
\hline Other & $13(13.3)$ \\
\hline \multicolumn{2}{|l|}{$\begin{array}{l}\text { How mHealth users learned about apps } \\
\qquad(\mathrm{n}=277)\end{array}$} \\
\hline Doctor/clinic & $27(9.8)$ \\
\hline Family/friend & $37(13.4)$ \\
\hline Website/Internet & $150(54.2)$ \\
\hline Flier/mail/other ad & $7(2.5)$ \\
\hline Other (eg, "app store") & $56(20.2)$ \\
\hline \multicolumn{2}{|l|}{$\begin{array}{l}\text { Importance for PCPs to know about } \\
\text { mHealth use }(\mathrm{n}=311)\end{array}$} \\
\hline Very important & $37(11.9)$ \\
\hline Important & $59(19.0)$ \\
\hline A little important & $93(29.9)$ \\
\hline Not at all important & $122(39.2)$ \\
\hline $\begin{array}{l}\text { My doctor has recommended a health app } \\
\quad(\mathrm{n}=322)\end{array}$ & $22(6.8)$ \\
\hline
\end{tabular}

Continued
Table 2. Continued

\begin{tabular}{lc}
\hline mHealth Use & $\begin{array}{c}\text { Primary Care } \\
\text { Patients } \\
\text { Reporting Use }\end{array}$ \\
\hline $\begin{array}{l}\text { How useful this feature would be on your } \\
\text { phone* }\end{array}$ & \\
Appointment reminders $(\mathrm{n}=318)$ & $4.0 \pm 1.4$ \\
Medication reminders $(\mathrm{n}=312)$ & $3.6 \pm 1.7$ \\
General health information $(\mathrm{n}=314)$ & $3.5 \pm 1.4$ \\
Track progress (eg, mood, weight) $(\mathrm{n}=$ & $3.5 \pm 1.5$ \\
$\quad$ 310) & $3.2 \pm 1.7$ \\
Help changing a habit (n $=308)$ & $3.2 \pm 1.6$ \\
Feedback on how I'm doing $(\mathrm{n}=312)$ & $3.2 \pm 1.7$ \\
Tell doctor how I'm doing $(\mathrm{n}=311)$ & $3.1 \pm 1.7$ \\
Stress management/coping $(\mathrm{n}=310)$ & $2.3 \pm 1.8$ \\
Support group/social network $(\mathrm{n}=308)$ & $1.9 \pm 1.8$ \\
Tell friend/family how I'm doing $(\mathrm{n}=$ & \\
$\quad$ 305) & \\
\hline
\end{tabular}

Data are number (\%) or mean \pm standard deviation.

*Usefulness of the features was rated on a scale of 0 (least useful) to 5 (most useful).

PCPs, primary care providers.

lowed closely by medication reminders, general health information, and health tracking, whereas social features such as support groups and updating friends or family were rated as less useful (Table 2).

Much of the patients' mHealth use occurred outside of the context of health care (Table 2). Fewer than $10 \%$ of respondents learned about mHealth apps from their doctor or clinic; only $6.8 \%$ of respondents reported that their physician had recommended a health app to them. Respondents did not feel it was important for their physicians to know about their use of health apps: Nearly $70 \%$ reported that this was "not at all" or "a little bit" important.

\section{Demographic and Clinical Correlates of Smartphone Ownership and Mobile Health Use}

In unadjusted models, older patients and those with a chronic medical condition were significantly less likely to own a smartphone than younger patients and those without chronic conditions. Latino patients were significantly more likely to own a smartphone than whites (Table 3). In multivariate analyses, age was significantly independently associated with smartphone ownership, whereas Latino ethnicity and chronic conditions were no longer significantly independently correlated. Similar findings emerged from analyses restricted to mobile phone owners (Online Appendix Table 1). 
Table 3. Correlates of Smartphone Ownership Among All Patients

\begin{tabular}{|c|c|c|c|c|c|}
\hline & \multicolumn{3}{|c|}{ Unadjusted Models } & \multicolumn{2}{|c|}{ Multivariate Model* $(\mathrm{n}=866)$} \\
\hline & Patients (n) & OR (95\% CI) & $P$ Value & Adjusted OR (95\% CI) & $P$ Value \\
\hline Age (years) & 809 & & & & \\
\hline $18-24$ & & Reference & & Reference & \\
\hline $25-34$ & & $1.13(0.65-1.95)$ & .68 & $1.13(0.65-1.98)$ & .66 \\
\hline $35-44$ & & $0.72(0.41-1.25)$ & .24 & $0.77(0.44-1.35)$ & .36 \\
\hline $45-54$ & & $0.36(0.21-0.60)$ & $<.001$ & $0.39(0.22-0.68)$ & .001 \\
\hline $55-64$ & & $0.18(0.10-0.30)$ & $<.001$ & $0.21(0.12-0.37)$ & $<.001$ \\
\hline$\geq 65$ & & $0.06(0.03-0.13)$ & $<.001$ & $0.08(0.04-0.17)$ & $<.001$ \\
\hline \multicolumn{6}{|l|}{ Sex } \\
\hline Male & & Reference & & Reference & \\
\hline Female & 811 & $0.87(0.63-1.21)$ & .41 & $0.75(0.51-1.09)$ & .13 \\
\hline Race/ethnicity & 818 & & & & \\
\hline White & & Reference & & Reference & \\
\hline Native American & & $0.89(0.33-2.45)$ & .83 & $0.78(0.26-2.39)$ & .66 \\
\hline Asian/Pacific Islander & & $1.25(0.56-2.79)$ & .58 & $0.74(0.3-1.83)$ & .51 \\
\hline African American & & $2.61(0.85-8.07)$ & .10 & $1.67(0.51-5.47)$ & .39 \\
\hline Latino & & $2.04(1.00-4.16)$ & .05 & $1.18(0.55-2.53)$ & .67 \\
\hline Other/multiracial & & $1.05(0.60-1.84)$ & .86 & $0.74(0.41-1.37)$ & .34 \\
\hline Any health literacy limitation & 797 & $0.87(0.65-1.17)$ & .37 & $0.86(0.62-1.2)$ & .38 \\
\hline Any chronic medical condition & 857 & $0.5(0.37-0.67)$ & $<.001$ & $0.74(0.53-1.04)$ & .08 \\
\hline Depression & 762 & $0.82(0.61-1.10)$ & .18 & $0.81(0.58-1.14)$ & .23 \\
\hline
\end{tabular}

All models were adjusted for clustering within clinics.

*The multivariate model reports results from a single model that includes all of the independent variables in the table. Multiple imputation was used for missing variables in the multivariate model.

CI, confidence interval; OR, odds ratio.

Like smartphone ownership, mHealth use was significantly associated with younger age in both unadjusted and multivariate models (Table 4), a pattern that was evident among analyses restricted to smartphone owners (Online Appendix Table 2). In unadjusted analyses, the presence of a chronic medical condition was associated with a significantly lower likelihood of mHealth use and Latino ethnicity was associated with a significantly higher likelihood of mHealth use, but neither pattern persisted in multivariate analyses or among analyses restricted to smartphone owners. Among smartphone owners, women were somewhat more likely than men to be mHealth users. Depression and health literacy were not associated with mHealth use.

\section{Discussion}

This study found comparable or greater rates of mHealth use among primary care patients than previously reported in the general population. Smartphone ownership among primary care patients $(55 \%)$ is approaching the penetration of In- ternet access among primary care patients a decade ago $(65 \%),{ }^{19}$ and our findings indicate that most patients with smartphones use mHealth tools. The extremely rapid adoption of mobile technologies among the general population is contributing to significant shifts in how people interact and how they seek information. This digital revolution is beginning to transform health care, presenting opportunities for improved service delivery as well as challenges for patients and providers who grapple with incorporating novel technologies into their daily lives and clinical workflows.

Our results, which suggest that patients may use mHealth to augment their use of formal clinical services, have important implications for health care providers. Of potential concern are the findings that few patients believe it is important for health care providers to know about their mHealth use. We did not assess reasons for this limited communication, and further research is warranted. Most mHealth users sought health information, and few used tools that were disease specific or otherwise connected to their health care providers. 
Table 4. Correlates of mHealth Use Among All Patients

\begin{tabular}{|c|c|c|c|c|c|}
\hline & \multicolumn{3}{|c|}{ Unadjusted Models } & \multicolumn{2}{|c|}{ Multivariate Model $^{*}(\mathrm{n}=879)$} \\
\hline & Patients (n) & OR $(95 \% \mathrm{CI})$ & $P$ Value & Adjusted OR (95\% CI) & $P$ Value \\
\hline Age (years) & 822 & & & & \\
\hline $18-24$ & & Reference & & Reference & \\
\hline $25-34$ & & $1.00(0.62-1.62)$ & .99 & $1.00(0.61-1.62)$ & .99 \\
\hline $35-44$ & & $0.64(0.39-1.05)$ & .08 & $0.66(0.4-1.1)$ & .11 \\
\hline $45-54$ & & $0.31(0.18-0.51)$ & $<.001$ & $0.32(0.19-0.55)$ & $<.001$ \\
\hline $55-64$ & & $0.14(0.08-0.25)$ & $<.001$ & $0.16(0.09-0.3)$ & $<.001$ \\
\hline$\geq 65$ & & $0.05(0.02-0.12)$ & $<.001$ & $0.07(0.03-0.16)$ & $<.001$ \\
\hline \multicolumn{6}{|l|}{ Sex } \\
\hline Male & & Reference & & Reference & \\
\hline Female & 825 & $1.28(0.92-1.79)$ & .14 & $1.15(0.79-1.66)$ & .47 \\
\hline Race/ethnicity & 831 & & & & \\
\hline White & & Reference & & Reference & \\
\hline Native American & & $1.33(0.50-3.52)$ & .57 & $1.16(0.4-3.34)$ & .78 \\
\hline Asian/Pacific Islander & & $1.11(0.52-2.37)$ & .79 & $0.72(0.31-1.66)$ & .44 \\
\hline African American & & $0.93(0.36-2.42)$ & .89 & $0.63(0.23-1.74)$ & .37 \\
\hline Latino & & $2.29(1.20-4.37)$ & .01 & $1.47(0.73-2.93)$ & .28 \\
\hline Other/multiracial & & $1.30(0.76-2.25)$ & .34 & $0.91(0.5-1.64)$ & .75 \\
\hline Any health literacy limitation & 810 & $1.00(0.74-1.33)$ & .98 & $0.98(0.7-1.36)$ & .89 \\
\hline Any chronic medical condition & 870 & $0.60(0.45-0.79)$ & $<.001$ & $0.91(0.66-1.26)$ & .58 \\
\hline Depression & 774 & $0.96(0.71-1.29)$ & .78 & $0.95(0.68-1.32)$ & .75 \\
\hline
\end{tabular}

All models are adjusted for clustering within clinics.

*The multivariate model reports results from a single model that includes all of the independent variables in the table. Multiple imputation was used for missing variables in the multivariate model.

CI, confidence interval; OR, odds ratio.

Given the type of tools now available, patients may not view physicians as a useful resource in advising them about mHealth tools and may prefer recommendations from their social network, peers, or other patients. Communication about mHealth tools may evolve as the tools themselves advance, with more tools allowing data-sharing with providers, or when health care systems offer mobile portals and apps directly to patients. However, other potential explanations warrant consideration. A recent report found that providers are suspicious of mHealth tools for patients; more than half of younger physicians have concerns that mHealth would foster too much patient independence, and nearly one quarter of such physicians discouraging use of mHealth tools. ${ }^{20}$ Lack of communication between health care providers and patients about mHealth use represents a missed opportunity for providers to support patients' self-management activities. ${ }^{9}$

Several findings have implications for the design and development of the next generation of mHealth tools and for their integration into health care systems. Consistent with research demonstrating that women use more health services and have greater health care help-seeking than men, ${ }^{21,22}$ we found that women reported somewhat greater mHealth use. If this pattern is replicated, considering how technology design may be able to reduce gender-based disparities in health care engagement would be important. Among our sample, mHealth use was not associated with race/ethnicity, health literacy, or depression, suggesting that mHealth tools may reach populations traditionally underserved in health care systems and thus may have a role in reducing pervasive disparities in care. As expected, smartphone ownership and mHealth use lag among older adults, although this gap will narrow as the younger generations of digital natives age and contribute their skills with technology and their expectations of its role to their interactions with health care providers.

Similar to the Pew mobile health survey, ${ }^{5}$ our unadjusted analyses revealed that mHealth use was less common among individuals with a chronic medical condition; however, this pattern did not 
hold in multivariate analyses. Thus, the apparent lower use of mHealth among individuals with chronic diseases may be related to other patient characteristics (such as older age) rather than the presence of a chronic disease per se, a distinction that is relevant in considering the potential reach of mHealth tools. For example, patients who develop chronic diseases at younger ages may experience particular benefit from technologies that support improved disease self-management to prevent future disability and complications; however, most of the tools that patients commonly use now do not target disease management, implying that future development should prioritize disease management tools that meet patients' needs. In contrast, tools intended for older adults should account for age in their design, for example, by offering large print interfaces or similar design elements, regardless of whether they are intended for patients with or without chronic diseases.

Most patients using mHealth tools were infrequent users, and many reported stopping use after a short time, most often because it took too much time to use, suggesting that existing tools may have limited appeal for patients managing chronic diseases that require self-management activities to be sustained over time. The application of patientcentered design methods would help ensure that tools are effective at meeting needs identified by patients. Concurrently, research examining how best to incorporate mHealth tools into clinical practice settings, including how to integrate them into clinical workflows, will be important for successful dissemination of tools in health care settings.

Several study limitations deserve mention. Data were obtained from a cross-sectional survey and, therefore, all information was based on self-report. The study was conducted in the northwest region of the United States and included predominantly white patients; therefore it is unclear to what extent the findings generalize to other regions or populations of different racial/ethnic composition. Potential selection bias could have occurred if respondents differed systematically from the general clinic population, for example, in their general or technology literacy. No data about patients who were offered the survey but declined were available. It is possible that some eligible patients were not offered the survey; however, we have no reason to suspect any systematic omissions that would con- tribute to selection bias. Although we cannot rule out the possibility of some selection bias, the overall response rate was high $(67.4 \%)$; the sample included patients across a wide range of ages, many of whom had health literacy limitations; and our findings share many patterns similar to those from the national Pew survey of the general population.

Our results support the notion that mHealth tools are rapidly becoming an acceptable and scalable means of empowering patients, yet health care providers play a limited role in assessing or advising patients on their use of mHealth tools. This finding is particularly notable because the 2 features that patients rated as most likely to be useful-appointment reminders and medication reminders-are directly related to their use of medical care, suggesting that patients may be open to using mHealth tools to enhance their relationships with health care providers. Although data are sorely needed to establish the evidence base for mHealth tools and interventions, health care providers who routinely ask their patients about their mHealth use may be especially effective at eliciting important information about patients' self-management activities, which may help these providers to be more adept supporting chronic disease care. To fulfill the promise for transforming health care service delivery that mobile health offers, however, the next generation of mHealth tools will need to align the capabilities of mHealth technologies with effective models of health care delivery by providing wellintegrated tools that address the needs of both patients and providers.

The authors thank the participating clinics, project champions, and patients for their valued contributions to this study.

\section{References}

1. Steinhubl SR, Muse ED, Topol EJ. Can mobile health technologies transform health care? JAMA 2013;310:2395-6.

2. Workman B. The explosion in health apps, and how they're disrupting the gigantic, lethargic health care industry. Business Insider, September 13, 2013. Availablefrom:http://www.businessinsider.com/mobile-willdisrupt-health-care-2013-9. Accessed September 25, 2014.

3. Pelletier SG. Explosive growth in health care apps raises oversight questions. AAMC Reporter, October 2012.Availablefrom:https://www.aamc.org/newsroom/ reporter/october2012/308516/health-care-apps.html. Accessed September 25, 2014. 
4. Wolf JA, Moreau JF, Akilov O, et al. Diagnostic inaccuracy of smartphone applications for melanoma detection. JAMA Dermatol 2013;149:422-6.

5. Fox S, Duggan M. Mobile health 2012. Washington, DC: Pew Research Center; 2012. Available from: http:// pewinternet.org/Reports/2012/Mobile-Health.aspx. September 25, 2014.

6. Fox S, Duggan M. Tracking for health. Washington, DC: Pew Research Center; 2013. Available from: http:// pewinternet.org/Reports/2013/Tracking-for-Health. aspx. Accessed September 25, 2014.

7. Mosa AS, Yoo I, Sheets L. A systematic review of healthcare applications for smartphones. BMC Med Inform Decis Mak 2012;12:67.

8. Donker T, Petrie K, Proudfoot J, Clarke J, Birch MR, Christensen H. Smartphones for smarter delivery of mental health programs: a systematic review. J Med Internet Res 2013;15:e247.

9. Lippman H. How apps are changing family medicine. J Fam Pract 2013;62:362-7.

10. Westfall JM, Zittleman L, Staton EW, et al. Card studies for observational research in practice. Ann Fam Med 2011;9:63-8.

11. Kroenke K, Spitzer RL, Williams JB. The Patient Health Questionnaire-2: validity of a two-item depression screener. Med Care 2003;41:1284-92.

12. Lowe B, Kroenke K, Grafe K. Detecting and monitoring depression with a two-item questionnaire (PHQ-2). J Psychosom Res 2005;58:163-71.

13. Chew LD, Bradley KA, Boyko EJ. Brief questions to identify patients with inadequate health literacy. Fam Med 2004;36:588-94.

14. Chew LD, Griffin JM, Partin MR, et al. Validation of screening questions for limited health literacy in a large VA outpatient population. J Gen Intern Med 2008;23:561-6.
15. Sarkar U, Schillinger D, Lopez A, Sudore R. Validation of self-reported health literacy questions among diverse English and Spanish-speaking populations. J Gen Intern Med 2011;26:265-71.

16. Sarkar U, Karter AJ, Liu JY, et al. The literacy divide: health literacy and the use of an internetbased patient portal in an integrated health system-results from the diabetes study of northern California (DISTANCE). J Health Commun 2010;15(Suppl 2):183-96.

17. Bauer AM, Schillinger D, Parker MM, et al. Health literacy and antidepressant medication adherence among adults with diabetes: the diabetes study of Northern California (DISTANCE). J Gen Intern Med 2013;28:1181-7.

18. Rubin D. Multiple imputation for nonresponse in surveys. New York: Wiley; 1987.

19. Schwartz KL, Roe T, Northrup J, Meza J, Seifeldin R, Neale AV. Family medicine patients' use of the Internet for health information: a MetroNet study. J Am Board Fam Med 2006;19:39-45.

20. Emerging mHealth: paths for growth. New York: PricewaterhouseCoopers; 2012. Available from: http:// www.pwc.com/en_GX/gx/healthcare/mhealth/assets/ pwc-emerging-mhealth-full.pdf. Accessed September 25, 2014.

21. National Center for Health Statistics. National Ambulatory Med Care Survey: 2010 summary tables. Atlanta (GA): Centers for Disease Control and Prevention; 2014. Available from: http://www.cdc.gov/nchs/data/ ahcd/namcs_summary/2010_namcs_web_tables.pdf. Accessed September 25, 2014.

22. Ashman JJ, Beresovsky V. Multiple chronic conditions among US adults who visited physician offices: data from the National Ambulatory Med Care Survey, 2009. Prev Chronic Dis 2013;10:E64. 
Appendix Table 1. Correlates of Smartphone Ownership Among Mobile Phone Owners

\begin{tabular}{|c|c|c|c|c|c|}
\hline & \multicolumn{3}{|c|}{ Unadjusted Models } & \multicolumn{2}{|c|}{ Multivariate Model $^{*}(\mathrm{n}=783)$} \\
\hline & Patients (n) & OR (95\% CI) & $P$ Value & Adjusted OR (95\% CI) & $P$ Value \\
\hline Age (years) & 738 & & & & \\
\hline $18-24$ & & Reference & & Reference & \\
\hline $25-34$ & & $1.15(0.60-2.20)$ & .67 & $1.14(0.6-2.17)$ & .69 \\
\hline $35-44$ & & $0.64(0.34-1.21)$ & .17 & $0.69(0.36-1.31)$ & .25 \\
\hline $45-54$ & & $0.27(0.15-0.49)$ & $<.001$ & $0.29(0.16-0.54)$ & $<.001$ \\
\hline $55-64$ & & $0.14(0.08-0.27)$ & $<.001$ & $0.16(0.09-0.31)$ & $<.001$ \\
\hline$\geq 65$ & & $0.05(0.02-0.11)$ & $<.001$ & $0.06(0.03-0.14)$ & $<.001$ \\
\hline \multicolumn{6}{|l|}{ Sex } \\
\hline Male & & Reference & & Reference & \\
\hline Female & 736 & $0.81(0.56-1.17)$ & .26 & $0.69(0.46-1.05)$ & .09 \\
\hline Race/ethnicity & 743 & & & & \\
\hline White & & Reference & & Reference & \\
\hline Native American & & $0.93(0.30-2.83)$ & .89 & $0.71(0.21-2.46)$ & .59 \\
\hline Asian/Pacific Islander & & $1.45(0.58-3.58)$ & .42 & $0.87(0.3-2.51)$ & .80 \\
\hline African American & & $2.07(0.67-6.41)$ & .21 & $1.27(0.38-4.26)$ & .70 \\
\hline Latino & & $1.95(0.90-4.20)$ & .09 & $1.05(0.46-2.43)$ & .91 \\
\hline Other/multiracial & & $1.26(0.66-2.37)$ & .48 & $0.86(0.43-1.73)$ & .67 \\
\hline Any health literacy limitation & 725 & $0.92(0.67-1.27)$ & .62 & $0.92(0.63-1.32)$ & .64 \\
\hline Any chronic medical condition & 776 & $0.56(0.41-0.77)$ & $<.001$ & $0.86(0.6-1.25)$ & .43 \\
\hline Depression & 690 & $0.84(0.61-1.15)$ & .28 & $0.79(0.55-1.16)$ & .23 \\
\hline
\end{tabular}

All models are adjusted for clustering within clinics.

*The multivariate model reports results from a single model that includes all of the independent variables in the table. Multiple imputation was used for missing variables in the multivariate model.

CI, confidence interval; OR, odds ratio. 
Appendix Table 2. Correlates of mHealth Use Among Smartphone Owners

\begin{tabular}{|c|c|c|c|c|c|}
\hline & \multicolumn{3}{|c|}{ Unadjusted Models } & \multicolumn{2}{|c|}{ Multivariate Model* $(\mathrm{n}=498)$} \\
\hline & Patients (n) & OR (95\% CI) & $P$ Value & Adjusted OR (95\% CI) & $P$ Value \\
\hline Age (years) & 473 & & & & \\
\hline $18-24$ & & Reference & & Reference & \\
\hline $25-34$ & & $0.83(0.43-1.59)$ & .58 & $0.83(0.42-1.61)$ & .58 \\
\hline $35-44$ & & $0.72(0.37-1.42)$ & .35 & $0.71(0.35-1.43)$ & .33 \\
\hline $45-54$ & & $0.41(0.21-0.83)$ & .01 & $0.43(0.21-0.89)$ & .02 \\
\hline $55-64$ & & $0.26(0.12-0.56)$ & .001 & $0.26(0.12-0.6)$ & .001 \\
\hline$\geq 65$ & & $0.17(0.05-0.57)$ & .004 & $0.19(0.05-0.68)$ & .011 \\
\hline \multicolumn{6}{|l|}{ Sex } \\
\hline Male & & Reference & & Reference & \\
\hline Female & 471 & $1.77(1.14-2.74)$ & .01 & $1.59(0.99-2.55)$ & .06 \\
\hline Race/ethnicity & 476 & & & & \\
\hline White & & Reference & & Reference & \\
\hline Native American & & $3.51(0.43-28.75)$ & .24 & $3.02(0.38-24.18)$ & .30 \\
\hline Asian/Pacific Islander & & $0.95(0.35-2.59)$ & .93 & $0.88(0.3-2.57)$ & .81 \\
\hline African American & & $0.44(0.15-1.29)$ & .13 & $0.36(0.11-1.1)$ & .07 \\
\hline Latino & & $1.77(0.70-4.44)$ & .23 & $1.43(0.55-3.73)$ & .47 \\
\hline Other/multiracial & & $1.49(0.65-3.38)$ & .35 & $1.21(0.51-2.85)$ & .67 \\
\hline Any health literacy limitation & 464 & $1.2(0.79-1.81)$ & .39 & $1.2(0.77-1.88)$ & .43 \\
\hline Any chronic medical condition & 493 & $0.95(0.64-1.41)$ & .81 & $1.2(0.77-1.88)$ & .41 \\
\hline Depression & 436 & $1.28(0.83-1.99)$ & .27 & $1.2(0.75-1.94)$ & .44 \\
\hline
\end{tabular}

All models are adjusted for clustering within clinics.

*The multivariate model reports results from a single model that includes all of the independent variables in the table. Multiple imputation was used for missing variables in the multivariate model.

CI, confidence interval; OR, odds ratio. 